\title{
MEDIA MIX MODELING COMPARISON OF INTERACTION MODEL TO SIMPLE LOG-LINEAR MODEL
}

\author{
PRIYANKA SHARMA*, JANAKI MEENA M, SYED IBRAHIM SP
}

School of Computer Science and Engineering, VIT University Chennai Campus, Chennai, Tamil Nadu, India. Email: psharma.sep@gmail.com Received: 23 January 2017, Revised and Accepted: 03 March 2017

\begin{abstract}
The objective of the current study is to compare a new model for media mix problem with popular model named as simple log-linear model. A modified approach proposed to improve the results of media mix model from simple log-linear method includes the simultaneous effect of different media variables on sales. The combined effect caused by various media variables shows a synergy in the curve for sales and hence considering it makes the model much effective and accurate.
\end{abstract}

Keywords: Media mix model, Simple log-linear model, Interaction model.

(C) 2017 The Authors. Published by Innovare Academic Sciences Pvt Ltd. This is an open access article under the CC BY license (http://creativecommons. org/licenses/by/4. 0/) DOI: http://dx.doi.org/10.22159/ajpcr.2017.v10s1.19763

\section{INTRODUCTION}

Marketing has always been the most significant component of gross expense by any company, and it is very important to know the real profit from the money invested in this area. In addition to traditional channels such as TV, radio, newspaper, and billboards companies now use many other channels too such as digital media and social media for marketing their products. With such spread types of expense, it becomes difficult to evaluate the benefit the company gains from each of these separately. The benefit of advertising by a particular way cannot be judged by a simple curve having a ratio of earning to expenditure since different kinds of products need different strategies, and each product faces a market of various heterogeneous customers [1,2]. Moreover, hence to provide a better allotment of expense to each of these channels, we use media mix modeling. Media mix modeling allows us to know the estimated future sales using a model built based on historical media data of investment. This whole set of data is designed to be used as a strategic tool for marketing planning and to help marketers get the maximum return on investment (ROI) return on their investments. To lead generation or to drive the sales or for enhancing the brand name, it is very usual for various marketing campaigns to run simultaneously or consequently on different offline and online media channels. A user who has made a purchase already or a user, who has changed the vendor, might have gone through several advertisements from various channels. To know what would be the maximal marketing coherence, every business person needs to ascertain that how a given budget should be allocated across channels to amplify business target measures such as revenue, ROI, lead generation or growth rate, and so on. Moreover, this can be done with the proper use of marketing mix modeling (MMM) [3].

Mathematically, media mix modeling has two parts or it can be implemented in any business with two steps. The first step includes identification of the response of some business target measures for spend value which is then the key and basis for the whole optimization. The second step includes the numerical optimization using any existing algorithm or some improvised algorithm. In this paper, we are taking simple log-linear algorithm as base and improvising it using interaction model. Existing algorithms are discussed briefly in literature survey section of the paper, and the proposed algorithm's idea has been given in section III. The later survey is concluded in the last section of the paper.

\section{LITERATURE SURVEY}

The implementation criteria for media mix model should be very simple, so there are basically four steps of implementing any media mix model which are discussed as below $[4,5]$ :

\section{Variable clustering}

This method is very often used in econometric studies. For example, a lot of brands assumed to effect the brand, for which the model is to be developed, and this influences MMM in a great way so considering these variables as "competition" is very important; also, market instruments are broadly classified as products, distribution, advertising, promotions, etc. Some other important variables are radio, newspapers, billboards, magazines, etc. If the data are available for sufficient period, then all these variables must be tested before beginning the next step which is an introduction to relative variables.

\section{Distinction of relative variable}

Statistical procedures such as principal component analysis and other methods for reduction of dimensions should be performed and also variables having high correlation should be checked and removed. Multiple appearances of highly correlated variables will affect the model making it inefficient. Furthermore, the variables such as income or spends should be converted to same units such as per capita and in constant dollars.

\section{Subproblem division of classes}

To get a wider assortment, we first solve the subproblems belonging to each product of similar brand and then summing up the model for whole brand. This enables the model to see each and every perspective of a single brand with multiple products belonging to different range. And also the division into subproblem enables a multidimensional view of the brand which helps in making the model personalized for it.

\section{Different level phasing of variables}

This can be accomplished by decomposition of variables which are dependent. Again the correlation can be used as a factor to decompose these variables. Demand models expect the variables to be present according to class in a different level. For example:

Revenue $=\mathrm{P} \times \mathrm{Q}$

Where,

$\mathrm{P}$ is price and $\mathrm{Q}$ is quantity.

And at the same time period and territory: 
$\mathrm{Q}=$ Number of buyer $\times$ average (size) per purchase $\times$ frequency of purchase.

\section{Value constraint on parameters}

Since this model is built for time series data, so there are multiple effects on variables overtime like lagging effect which is further discussed in the paper. Some decay constants are applied on data variables to maintain the effectiveness of the variable.

After processing the above steps, we need to predict spends and this intend response to spend can be approximated generally and broadly in two ways:

- Top-down approach

- Bottom-up approach.

Bottom-up approach includes building attribution model created at user level (Shao and Li, 2011) [6] applied to each and every conversion and then finally sum of overall conversion gives contribution for each channel. However, to establish an authentic, dependable, and efficient attribution, model could be acutely challenging because there are hundreds and thousands of factors that are needed to be considered. Hence, the first and foremost task to be performed is clustering of these media variables into subcategories such as "time series variables," "gross rating point (GRP)," and "click rate" also channel correspondence and user deed related attributes, for example, all the variable such as discount, promotions and offers will come into one group and then channel's TRP and GRP will certain category which will ultimately sum up to final categories of attributes to know the variables which are significant for our process of media mix modelling.

While on the other hand, top-down approach disregards all the details at user level and it directly focuses on spend by each and every media channel which brings many problems such as perpetuated or lagging effect of any advertisement on user's conversion behavior; this problem is usually termed as advertising adstock [7] or carryover effect (Broadbent, 1979). There are various kinds of lagging effects as stated below [8]:

- Execution delay:

This is the delay caused between management spending money and preparing the advertisement.

- Nothing delay:

The time interval between the advertisement being published and read by user is nothing delay. Moreover, it is very important to consider this in the model for efficiency.

- Purchase delay:

The time between when the customer receiving the stimulus to purchase the product and the purchase actually being made is known as purchase delay.

- There is also customer holdover effect which comes in existence when a new buyer is found or when a new buyer encounters the product, so this holdover effect makes him change the sales effectively in the initial period [9].

In bottom-up approach, this problem can be handled using time variables from user's history, but for top-down approach, we need to include the adstock factor to our equation used in the algorithm. Now, this addition of adstock factor brings another problem which is a change in actual numbers and hence then the data are needed to be passed through another process known as transformation for scaling. The existing process followed generally is shown as in Fig. 1 [10].

Simple log-linear regression is used with all the variables passed after transformation step. In transformation, the equation used to scale the variables is shown as Equation (1) [11], and the scaled resulted variables are then put into the equation of simple log-linear model as shown in Equation (2) [12,13].

$X(t)=K_{\exp }\left(\log \left(\frac{X(0)}{K}\right) \exp (-\alpha t)\right.$
Where,

$\mathrm{X}(0)$ is the advertising value at the start.

$\mathrm{K}$ is the positive coefficient.

$\alpha$ is another constant.

$\exp =\left(c+\sum_{i} w_{i} f_{i}(X)\right)$

Where,

$\mathrm{w}_{\mathrm{i}}$ stands for various parameter values used from different variables.

$A_{t}=T_{t}+\lambda A_{t-1} \quad t=1, \ldots, n[5]$

Above is the adstock equation where

$\lambda$ as the lag parameter.

At as calculative advertisement effect and $\mathrm{T}_{\mathrm{t}}$ is effect of advertisement effect at time $\mathrm{T}$.

One more approach which is popular is conjoint analysis is basically a reverse analysis and it helps to know various features or key products responsible for sales. This analysis involves methods such as Hierarchical Bayes estimation, logit methodology, and regression. The methodology which we are considering is an extension of log-linear regression with integration of some variables as explained in the next section of the paper.

Adaptive model building: There is a broad division for forecasting done by media mix models [14]:

- Unconditional forecasting

- Conditional forecasting.

The major concern while building any MMM model is the market keeps changing and so is the behavior of consumers; thus, it is not possible to take media model building as a onetime affair. Brand's product may change overtime and also the group of consumer may increase or decrease with time. Hence, modification in product characteristics should be kept dynamic while building the model so that the update and integration of model becomes easier. With the requirement of brand holder, there may have some changes in brand-level parameters too. Hence, the hard-coded model would create a problem and hence general approach should be followed. All observable market changes should be kept in middle while building the model so as to keep the effectiveness of MMM even when market changes. Non-robust models are very difficult to deal with when changes are to be made.

\section{PROPOSED SYSTEM}

Although the usual approach of media mix model as discussed in above section gives considerable results but to enhance the results for more accurate forecast of spends on media we need to consider the relative effect of one variable on other. The variables we take in model are used as mutually exclusive to each other while they can have a considerable effect on the model if taken otherwise. And hence interaction model comes into existence.

Interaction model basically takes the combined effect of two variables on the model, and in the case of media mix modeling, we have various media channels and their effect on consumers so if we take effect of two variable separately at a certain time, then we miss the combined and relative effect of these variables on consumer. So this effect is known as interactive effect and it gives a synergy curve as it has smaller inflection point and sudden rise as shown in Fig. 2 [15]. This can be understood by equations below:

$\mathrm{Y}_{\mathrm{i}}=\mathrm{B}_{\mathrm{o}}+\mathrm{B}_{0} \mathrm{X}_{\mathrm{i}}$

$\mathrm{Y}_{\mathrm{i}}=\mathrm{B}_{\mathrm{o}}+\mathrm{B}_{\mathrm{o}} \mathrm{Y}_{\mathrm{i}}$

$\mathrm{Y}_{\mathrm{i}}=\mathrm{B}_{0}+\mathrm{B}_{0} \mathrm{X}_{\mathrm{i}} \mathrm{Y}_{\mathrm{i}}$ 


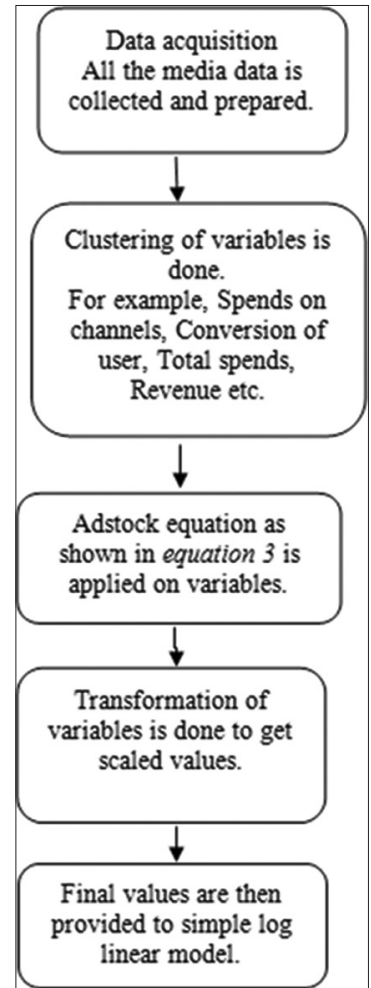

Fig. 1: Existing general process for marketing mix modeling

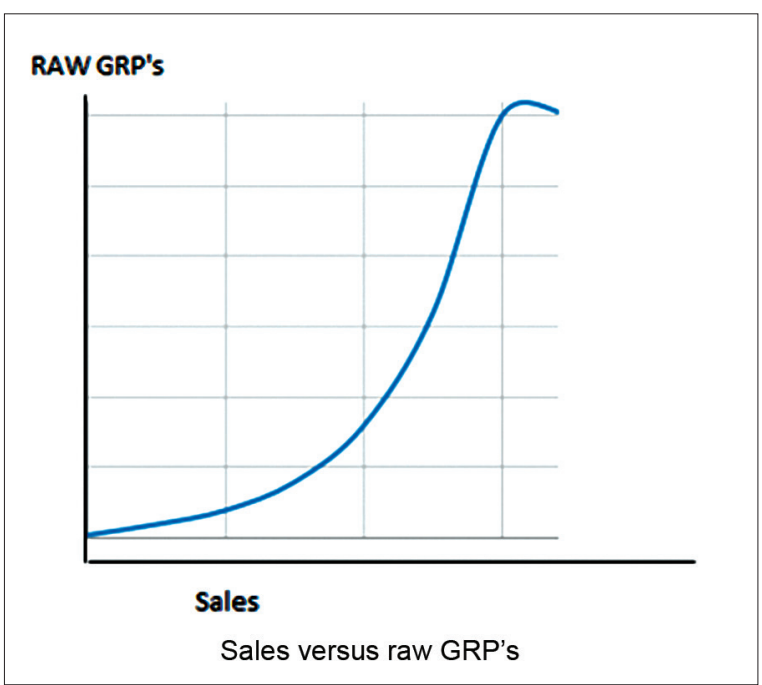

Fig. 2: Synergy effect shown in curve caused by taking combined variables

In Equations (4) and (5), we see the separate effect of media variables on $Y_{i}$ while the interaction model considers Equation (6) as well which shows the curve given in Fig. 2.

The curve shown in Fig. 2 is known as synergy curve, and this shows the combined effect of variables on users. When used as combined variable the graph of sales versus raw GRPs goes exponentially up and them with time and lagged effect, the curve shows variation toward the original axis.

Synergy curves show the exponential heights when a highly effective parameter is added to the attributes and hence in Fig. 2, when more than one parameter or attributes are taken into consideration, it shows a great height of sales and spends increases exponentially in that period of time.
Hence, the proposed approach involves consideration of combined effect by media variables rather than taking them in mutually exclusive manner while preparing the model. Furthermore, the combined effect should be taken in such a manner that every set of variables having low correlation and high impact on sales is considered. The relative and conditional probability is taken while making the model of all these variables over each other, and this enables the model to predict actual sales. Model building may include either multinomial, logistic, or any other approach but the steps before implementing these equations for hypothesis are very important, and most of the effective time should be spent on parameter decision. The conclusion of the proposed approach and its impact on model is discussed in the next section of this paper.

\section{CONCLUSION}

There are certain factors to decide whether a media mix model is appropriate or not. These factors include the recent forecast and actual data match from any data source provider which collects data from market survey. Furthermore, the important thing to see the effectivity of model fairly is to match the variables which were taken while building the model and while doing the survey should be same otherwise the results forecasted would not match with survey results. Finally, when we compare and evaluate the complete and comprehensive sample performance of our model with the various forms of the multinomial logistic regression model where we also checked and rectified for every possible heterogeneity from this result, we can easily deduce that while using the smallest amount of parameters, our model surpasses all the variants on forecasting. This, together with the face validity of our parameter results, leads us to believe that incorporating responsiveness seems to be a worthwhile exercise. Every single response from smallest of attribute contributes a lot to the model so considering the right set of variables is the main task while building the model and also to keep a check on the effect of each variable on other variable bring efficiency to the model.

Practically, whenever an advertisement is put on billboard, it has a lesser effect on sales as compared to when it is put with a discount mentioned on it. Hence, the combined effect of discount in the same period when advertisement is out for users causes a significant hike in sales in for the same period. In general, when we take effect of advertisement's value and discount's value separately, then the predicted value of sales is lower than the original sales value in that period because the sales are high due to combined effect and hence the values are not predicted efficiently. Hence, using interaction model, we can overcome this problem and enhance our media mix model. In this survey paper, we have proposed a system in which multiple variables are being combined and provided to the model so that the synergy effect caused due to the interaction of these variables is noticed and hence provides the actual view of sales in the particular period. Interaction of variables also includes many clusters as to what combination of variables is effective and making an impact on sales. And hence only those clusters should be included in model rather than all the combinations.

Hence, this should be very clear while building a model that the selection strategy leads to an experiential and observational model with improved in and out of sample fit performance. Furthermore, since we have to take care of market and keep the model consistent for maintaining the share attraction, we keep the model equations of reduced form generally linearly dependent so as to maintain the firmness of it. This clearly suggests us that the parameter interpretation is very important in estimation procedure of model. Another thing to be considered is that the self-evident and the permanent effect of a temporary promotion is often considered as interesting, vigorous, and dynamic feature for any model, so even while taking distribution variables, we should consider the effect of these time series variables such as promotional events or holiday sales. Many ad hoc methods are available for building this media mix model, but each and every method would agree to the interaction approach provided in this paper. Since it is a general idea and can be integrated in any technique. Hence, the 
modeling could be regression with log-linear equation or polynomial regression, but the variables taken in equation should be according to the interaction model for making it effective and better.

\section{REFERENCES}

1. Aaker DA. Building Strong Brands. New York, NY: Free Press; 1995.

2. Cain PM. Dynamic Marketing Mix Modelling and Digital Attribution. US: Market Science Consulting; 2014.

3. Cain PM. Integrated brand marketing and measuring returns. Marketing Mix Modeling and Return on Investment. Basingstoke: Market Science Consulting; 2010.

4. Raftery AE, Dean N. Variable selection for model-based clustering. J Am Stat Assoc 2006;101(473):168-78.

5. Green PE, Krieger AM. Alternative approaches to clusterbased market segmentation. J Mark Res Soc 1995;37(3):221-39.

6. Liu Y, Laguna J, Wright M, He H. Media mix modeling - A monte carlo simulation study. J Mark Anal 2014;2(3):173-86.

7. Leone RP. Generalizing what is known about temporal aggregation and advertising carry-over. Mark Sci 1995;14(3):141-50.

8. Alcaraz R. Conceptual reasons beyond technology for the delayed progress and innovation in marketing science. Appl Mark Anal 2014; $1: 21-31$

9. Ambach G, Hess M. Measuring long term effects in marketing. Mark Res Summer 2000: 22-7.

10. Dash M, Andrews SB. A Study on Market Behaviour and Price Discovery in Indian Commodity Markets; 2010. Available from: https:// www.ssrn.com/abstract=1722770; http://www.dx.doi.org/10.2139/ ssrn. 1722770

11. Chu WL, Wu FS, Kao KS, Yen DC. Diffusion of mobile telephony: An empirical study in Taiwan. Telecomm Policy 2009;33(9):506-20.

12. Logan JD. Applied Mathematics-A Contemporary Approach. Hoboken: John Wiley and Sons; 1987.

13. Mahajan V, Muller E, Bass FM. New product diffusion models in marketing: A review and directions for research. J Mark 1990;54(1):1-26.

14. Taylor N. The economic value of volatility forecasts: A conditional approach. J Financ Econ 2013;12(3):433-78.

15. Shao X, Li L. Data-driven multi-touch attribution models, KDD'11. In: Proceedings of the $17^{\text {th }}$ ACM SIGKDD International Conference on Knowledge Discovery and Data Mining; 2011. p. 258-64. 